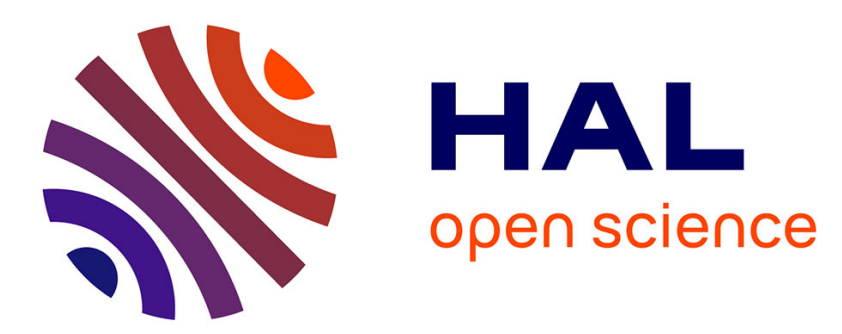

\title{
Using prompts to produce quality corpus for training automatic speech recognition systems \\ Benjamin Lecouteux, Georges Linarès
}

\section{To cite this version:}

Benjamin Lecouteux, Georges Linarès. Using prompts to produce quality corpus for training automatic speech recognition systems. MELECON 2008 - The 14th IEEE Mediterranean Electrotechnical Conference, May 2008, Ajaccio, France. 10.1109/MELCON.2008.4618540 . hal-01318050

\section{HAL Id: hal-01318050 \\ https://hal.science/hal-01318050}

Submitted on 9 Nov 2017

HAL is a multi-disciplinary open access archive for the deposit and dissemination of scientific research documents, whether they are published or not. The documents may come from teaching and research institutions in France or abroad, or from public or private research centers.
L'archive ouverte pluridisciplinaire HAL, est destinée au dépôt et à la diffusion de documents scientifiques de niveau recherche, publiés ou non, émanant des établissements d'enseignement et de recherche français ou étrangers, des laboratoires publics ou privés. 


\title{
Using prompts to produce quality corpus for training automatic speech recognition systems
}

\author{
Benjamin Lecouteux and Georges Linarès
}

\begin{abstract}
In this paper we present an integrated unsupervised method to produce a quality corpus for training automatic speech recognition system (ASR) using prompts or closed captions. Closed captions and prompts do not always have timestamps and do not necessarily correspond to the exact speech. We propose a method allowing to extract quality corpus from imperfect transcript. The proposed approach works in two steps. During the search, the ASR system finds matching segments in a large prompt database. Matching segments are then used inside a Driven Decoding Algorithm (DDA) to produce a high quality corpus. Results show a F-measure of $96 \%$ in term of spotting while the DDA corrects the output according to the prompts: a high quality corpus is easily extracted.
\end{abstract}

Index Terms - speech recognition, closed captioning, corpus building, automatic segmentation

\section{INTRODUCTION}

The training of an automatic speech recognition system (ASR) requires large amounts of exact annotated speech. The transcription task is expensive and takes a lot of time. In some situations imperfect transcripts like journalist prompts, closed captions or abstracts are available. This material is available in large quantities.

However, these transcripts present two issues: the distance compared to the audio stream and the lack of timestamps. Various approaches propose to use imperfect transcripts for unsupervised ASR training (section II-D). But existing methods are not integrated and have shortcomings: processes are iterative and take a lot of computing time; the lack of timestamp is forgotten. Moreover existing methods do not use all the potential of imperfect transcripts.

The first part of this paper is dedicated to the related work on these issues: the prompts quality, methods to perform ASR alignment with imperfect transcripts, the automatic imperfect transcript segmentation, and finally how to use them for training an ASR system.

In a second part, we describe an integrated approach allowing us to solve the two main approximated transcription issues:

- In section III-A we describe the driven decoding algorithm which allows us to drive an ASR according to a transcript. DDA allows to correct on the fly an approximated transcript.Then we present some DDA experiments, results and the DDA ability to exploit dynamically imperfect transcripts.

\footnotetext{
${ }^{1}$ This research is supported by the ANR (Agence Nationale de la Recherche), AVISON project.

B. Lecouteux and G. Linarès are with the Laboratoire Informatique d'Avignon (LIA), University of Avignon FRANCE (e-mail : benjamin.lecouteux, georges.linares@univ-avignon.fr)
}

- The section III-B presents the "spotting text island algorithm" to select segments in real-time into a large prompt database. This algorithm allows to synchronize prompts with the ASR system. The experiments are carried out on the RTBF and ESTER databases.

The section III-C presents the text spotting integration into the driven decoding algorithm. We discuss how this method makes it possible to build high quality corpora. Finally the last section presents the conclusions and future works.

\section{RELATED WORK}

\section{A. Quality of prompts or closed captions}

P. Cardinal presents in [1] the most common problems with journalist prompts: journalist stories associated to speech are imperfect. Prompts are not always respected, sentences are forgotten or inserted, and other sentences are pronounced with some word variations because texts are often just a guideline for the journalist. In the case of closed captions the WER are $10 \%$ to $20 \%$ compared to the exact transcript [2]. Experiments computed by M.J. Witbrock [3] have shown that approximately $16 \%$ of the words are incorrectly transcribed compared to the exact transcript. Moreover he shows that using transcripts $a$ posteriori is not always relevant: experiments with a trigram language model derived from a correct transcript showed a high word error rate.

\section{B. Automatic closed caption alignment}

Different methods are proposed to align closed-captions with the output of an ASR. In [4], P.J. Moreno \& al. propose to align long audio documents with their exact transcripts within the framework of automatic indexing of multimedia documents. His method is based on the search of anchor points which are isolated from the extracted segments with a high correlation between the a-priori transcript and the automatic transcript. However this method is only applicable to low-error texts.

In [1] P. Cardinal \& al. aligns ASR outputs with imperfect texts by computing their edit-distance which is the minimal cost to obtain a final sentence. To compute minimal cost, M. Mohri uses FSTs [5]. The two strings are represented by transducers $T_{\text {output } A S R}$ and $T_{\text {closedcaption }}$. An edition funtion $T_{\text {edit }}$ is associated to each closed caption. Then the set of all alignments is computed by :

$$
T_{\text {aligns }}=T_{\text {outputASR }} \oplus T_{\text {edit }} \oplus T_{\text {closedcaption }}
$$

The best path is computed by performing a best path search : 


$$
\text { BestPath }=B P S\left(T_{\text {aligns }}\right)
$$

This operation is performed for each potential closedcaption. A module rejects bad alignments. Good alignments are used for training acoustics models. However this is an $a$ posteriori method: more than one pass must be performed and the alignment is heavy in terms of calculation time. Moreover bad decoded segments can not be aligned.

In [3], M.J. Witbrock uses timestamps and aligns matching closed-captions with classic dynamic time warping (DTW, [6]) algorithm. However this is a batch method with the same limitations that method proposed in [1].

P. Placeway proposes a more integrated method in [2]. He proposes a translation model for mapping caption sequences to word sequences which updates the language model. The translation model (figure 1) is a Markov chain where arcs represent deletions, insertions or substitions: this model corresponds to the string edit distance with a DTW. The model is integrated in the decoder but timestamps are essential to select the good caption model. These experiments are carried out using a synchronous decoder (Sphinx-3, [7]).

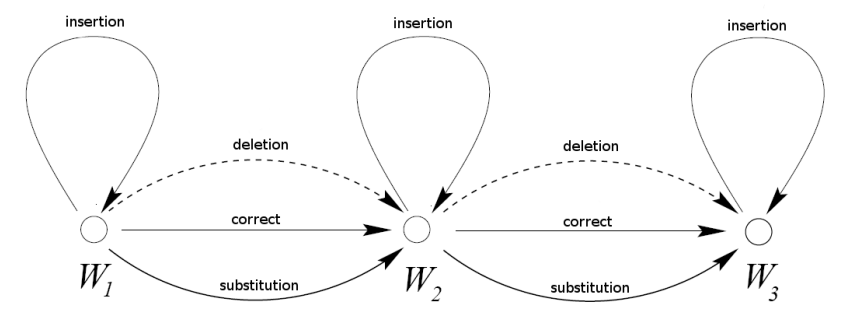

Fig. 1. The translation model (a Markov chain) where arcs represent deletions, insertions or substitions

Previous methods are limited to low WER transcripts. In a recent paper A. Haubold proposes an approach for the alignment of speech to highly imperfect transcripts in [8]. His approach is similar to the previous, but temporally unaligned text is converted to phonemes. Then the alignment is performed by using an edit-distance similar to the alignment between two DNA sequences. This obtained promising results.

\section{Automatic segmentation on partial transcriptions}

The goal of segmentation is to select the segments in a (large) prompt database which match the current speech. In [9] the authors correct the approximate time marks from the closed-caption transcriptions. They align the closed-caption with the automatic audio transcription. Then the algorithm assigns timestamps to closed captions according to the automatic transcription. However, in this approach, time marks are available in closed captions. The work presented in [10] also uses available closed-captions with their boundaries.

In [1], P.J. Moreno proposes a method allowing the selection of segments in a large database. A disadvantage of this approach is the computing time: all potentials segments must be tested. This approach presents a segmentation accuracy of $80 \%$ with a false reject rate of $30 \%$.

Some algorithms adapted to find a local segmentation have been developed: the Smith-Waterman [11] algorithm which is designed to perform local sequence alignment; originally for determining similar regions between two nucleotide or protein sequences. The Smith-Waterman algorithm compares segments of all possible lengths and optimizes the similarity measure to find sub-sequences. However the algorithm requires a lot of time and memory.

The problem can be tackled as textual information retrieval. In text retrieval, the problem is to find documents meeting the user's information need. The vector model is the most used model for information retrieval. With this model documents are represented in a space $D$ whose dimensions are the words composing the documents. Words are extracted from the documents after stripping stop words and stemming them [12]. Queries are represented in the same space as documents, like the documents. The $t f \times$ x $i d f$ (term frequency $\mathrm{x}$ inverse document frequency) is often used to get an estimation of the information carried by a word [13]. The similarity between queries and documents are computed (The cosine similarity is widely used : it is the cosine of the angle between the document vector and the query vector) and documents ranked by the similarity measure. This operation is fast (section IIIB) and allows us to retrieve a matching segment from a large document.

\section{Using prompts for training an ASR}

One of the main interest in improving corpus quality is the training of acoustic models for ASR systems.

In [10], L. Lamel proposes a recursive method for acoustic model training using low quality transcribed databases. This approach consists of three steps :

- Decode the training database automatically.

- Find matched segments between approximated transcripts and automatic decoding.

- Matching segments are used for acoustic model reestimation.

This method was tacked by M.J. Witbrock [3]. He uses teletext/closed-caption for re-training acoustic models based on television input. The ASR is used to find matching closedcaptions. These segments are then used to retrain acoustic models. In [14] the authors investigate the use of closedcaptions for MMI (Maximum Mutual Information) or MPE (Minimum Phone Error) discriminative training with a similar scheme.

Another approach proposed by P. Placeway [2] is to estimate a language model with prompts or closed-captions. The estimated model is interpolated with a generic language model in the ASR. This technique improves the results, but some subtitles information is drowned in the data quantity. Moreover, P. Placeway included a closed caption model into the beam search: words matching the imperfect transcript are favored. This method is limited to closed-captions with timestamps. 
In [15], the authors propose a light supervision method to acquire acoustic training data from speech having corresponding prompts. They estimate a biased language model using imperfect transcripts. The ASR output is aligned to the approximated transcripts and only matching words are selected for acoustic training. They obtain $13 \%$ relative error rate reduction with 702 hours added to the baseline (141 hours of training data). However the partial imperfect transcript information is not directly included in the decoder.

\section{APPROACH}

Our objective is to exploit imperfect transcripts like prompts or closed-captions when no timestamp information is available. The proposed method is integrated: matching segments are selected on the fly in a large database by the ASR system. Once matching segments are selected, they drive the ASR system.

Firstly we present the driven decoding algorithm (DDA) and secondly the fast-match to transcript island.

\section{A. The Driven Decoding Algorithm}

Previously, we proposed a Driven Decoding Algorithm (DDA) which is able to simultaneously align and correct the imperfect transcripts [16]. DDA works with SPEERAL [17], an asynchronous decoder derived from the $A^{*}$ algorithm. The ASR generates assumptions as it progresses the phoneme lattice. For each new step, the current assumption is aligned with the approximated transcript (figure 2). Then a matching score $\alpha$ is computed and integrated with the language model:

$$
\tilde{P}\left(w_{i} \mid w_{i-1}, w_{i-2}\right)=P^{1-\alpha}\left(w_{i} \mid w_{i-1}, w_{i-2}\right)
$$

where $\tilde{P}\left(w_{i} \mid w_{i-1}, w_{i-2}\right)$ is the updated trigram probability of the word $w_{i}$ knowing the history $w_{i-2}, w_{i-3}$, and $P\left(w_{i} \mid w_{i-1}, w_{i-2}\right)$ is the initial probability of the trigram.

When the trigram is aligned $\alpha$ is at a maximum and decreases according to the misalignments of the history (values of $\alpha$ are determined empirically using a development corpus).

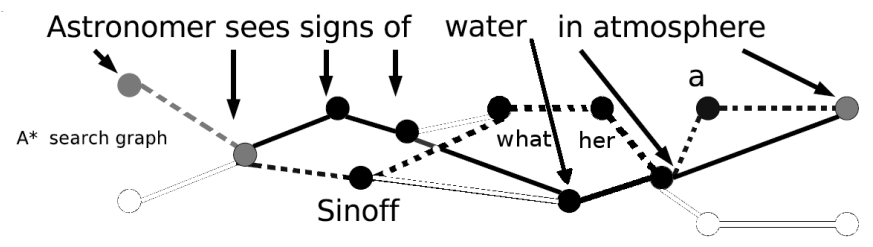

final path with DDA

$\ldots \ldots$ origin final path $\quad$ joint path

Fig. 2. The DDA mechanism drives the search by dynamically rescoring search function according to the alignment scores.

1) Experiments: In the first experiment, approximated transcripts are already segmented. The alignment is integrated into the ASR, and based on the Swith-Waterman algorithm. DDA experiments were carried out with the "Broadcast news" system developed by the LIA for the French evaluation campaign ESTER [18]. The system is assessed on 3 hours of radio broadcast extracted from the ESTER development corpus (France Inter 1, France Inter 2, France Info). Imperfect transcripts are made by adding errors manually in the exact transcript: $10 \%$ WER in France Inter shows, and 20\% WER in France Info show.

2) Alignment with exact transcripts: Preliminary experiments with exact transcripts allow us to evaluate the DDA potential (table I). The baseline system is $22.7 \%$ WER. Using a language model estimated on the transcript reduces the WER to $5.2 \%$. However better results are found when using alignment during the graph search (section III-A): $3.7 \%$ of WER. Our experiments show that using the estimated language model with the dynamic alignment does not improve the system. The minimal WER for a method re-estimating the concurrent hypothesis without modifying the content of the hypothesis stack is $3.7 \%$.

\begin{tabular}{|l|l|}
\hline & WER \\
\hline FrInter 1: ML-G alone & $22.7 \%$ \\
\hline FrInter 1: ML-TrEx alone & $5.2 \%$ \\
\hline FrInter 1: ML-G + ML-TrEx & $10.8 \%$ \\
\hline FrInter 1: ML-G alone + alTrEx & $3.7 \%$ \\
\hline FrInter 1: ML-TrEx alone + alTrEx & $3.7 \%$ \\
\hline FrInter 1: ML-G + ML-TrEx + alTrEx & $3.7 \%$ \\
\hline
\end{tabular}

TABLE I

EXPERIMENTS WITH EXACT TRANSCRIPT : ML-G IS THE GENERIC LANGUAGE MODEL, ML-TEEX IS THE LANGUAGE MODEL ESTIMATE ON THE PERFECT TRANSCRIPT AND ALTREX IS AN DYNAMIC ALIGNMENT TO THE PERFECT TRANSCRIPT DURING THE GRAPH SEARCH

3) Alignment with imperfect transcripts: Experiments with approximated transcripts show the same behavior (table II). Interpolated language models improve the baseline, but temporal information is lost. Using dynamic alignment, imperfect transcripts drive the decoder, and reciprocally, the decoder corrects the imperfect transcripts.

\begin{tabular}{|l|l|}
\hline & WER \\
\hline FrInter 1: ML-G alone & $22.7 \%$ \\
\hline FrInter 1: ML-TrErr alone & $16.3 \%$ \\
\hline FrInter 1: ML-G + ML-TrErr & $15.2 \%$ \\
\hline FrInter 1: ML-TrErr + alTrErr & $9.9 \%$ \\
\hline FrInter 1: ML-G + alTrErr & $7.7 \%$ \\
\hline FrInter 1: ML-G + ML-TrEr + alTrErr & $7.2 \%$ \\
\hline
\end{tabular}

TABLE II

INTERPOLATION OF THE GENERIC LANGUAGE MODEL (ML-G) WITH THE MODEL TRAINED ON THE IMPERFECT TRANSCRIPT (ML-TRERR - 10\% WER)

4) Final results with DDA: In order to validate these results, we tested the system on a larger corpus. Two hours are processed using the same evaluation protocol described in the last sections. We observe that the gain in performance seems to be relatively independent from the quality of the initial transcript (table III).

These experiments show that the DDA is able to correctly align imperfect transcripts during the search. Moreover DDA dramatically improves the initial transcript: any imperfect information is exploited and corrected. Contrary to a-posteriori alignments DDA directly improves the decoder quality. 


\begin{tabular}{|l|l|l|l|}
\hline Shows & Baseline & Transcript & TDS \\
\hline France Inter 1 & $22.7 \%$ & $10.1 \%$ & $7.2 \%$ \\
\hline France Inter 2 & $21.1 \%$ & $10.2 \%$ & $7.7 \%$ \\
\hline France Info & $24.3 \%$ & $20.3 \%$ & $12.1 \%$ \\
\hline
\end{tabular}

TABLE III

WER OBTAINED BY THE BASELINE SYSTEM (Baseline), WER IN THE ORIGINAL TRANSCRIPT (Transcript), WER OBTAINED BY TRANSCRIPT DRIVEN SYSTEM $(T D S)$

\section{B. Spotting transcript-Islands}

Previously, the DDA algorithm was not able to align large segments in reasonable time. The Swith-Waterman algorithm complexity is $O(n, m)$ with $n$ the number of query terms and $m$ the number of document terms. In order to apply DDA to transcript-island spotting we add a fast-match process which aims to find on-the-fly the transcripts segments which are relevant to the current state of the search algorithm [19].

1) Fast-match transcript-island: The principle of the text island spotting (figure 3) is close to approaches used in the field of information retrieval. In our case, the hypothesis is a query searching a transcript-island. Search engines try to find the most relevant documents by comparing the query to the indexed collection of stored documents. As the current hypothesis is developped, a set of word clusters are built or updated. Clusters are the intersection between query and the large database. An adapted similarity measure is performed on each cluster and the most relevant are selected for alignment (based on the score being greater than a fixed a-priori threshold).

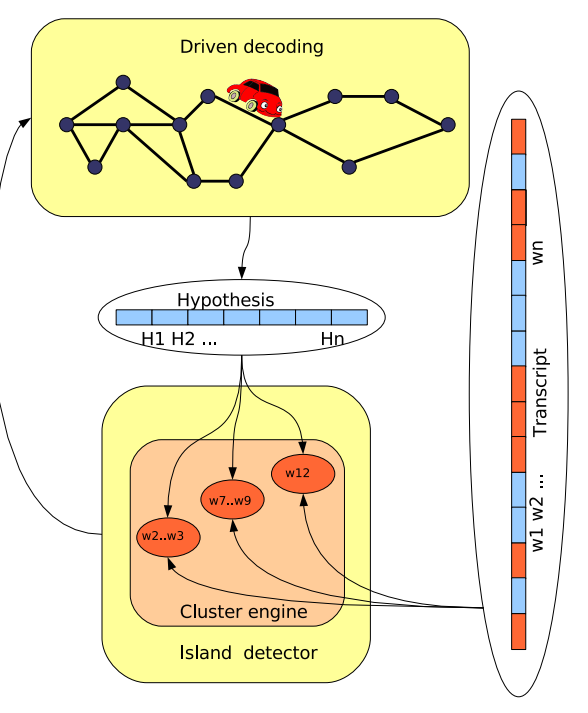

Fig. 3. Scheme of principle of spotter integration to Driven decoding. Transcripts-islands are detected by the spotter which computes an assumptionto-island matching score. According to it, the spotter sets the decoder in driven decoding mode.

2) Spotting exact transcript island on ESTER database: In the first experiment, we have tested the spotting algorithm with exact transcript. Moreover we have removed $50 \%$ of the transcription to test the spotting quality. Experiments are assessed on three hours extracted from the ESTER development corpus (France INTER, France INFO, RFI). During the search each assumption is presented as a query: the spotting algorithm proposes a corresponding segment in the database. The table IV presents the results. With perfect transcripts, the F-measure is $95.5 \%$.

\begin{tabular}{|l|l|l|l|l|}
\hline $\begin{array}{l}\text { Radio } \\
\text { station }\end{array}$ & Precision & Recall & F-measure & $\begin{array}{l}\text { Seg. } \\
\text { number }\end{array}$ \\
\hline FrInter & $90.7 \%$ & $96.9 \%$ & $93.7 \%$ & 478 \\
\hline FrInfo & $93.4 \%$ & $89.7 \%$ & $91.5 \%$ & 468 \\
\hline RFI & $98.8 \%$ & $97.8 \%$ & $98.4 \%$ & 812 \\
\hline Mean & $95.3 \%$ & $97.3 \%$ & $95.5 \%$ & 1758 \\
\hline
\end{tabular}

TABLE IV

SPOTTING ON THE ESTER DATABASE. EXPERIMENTS ARE PERFORMED ON 3 HOURS OF THE DEVELOPPEMENT SET, BY USING IMPERFECT TRANSCRIPTS OF ABOUT 10\% WORD ERROR RATE. AS IN TABLE 1, 50\% OF TRANSCRIPT SEGMENTS HAVE BEEN REMOVED FOR SPOTTING EVALUATION.

3) Spotting imperfect transcripts on ESTER database: In the second experiment, we have added errors into the transcripts $(10 \%)$ to test the robustness of the presented spotting algorithm. Results are similar to the previous experiment (table V). In the table results on real condition with RTBF (Radio Télévision Belge Francophone, a Belgium broadcast news radio) database are added. This implies the algorithm is able to quickly find segments in spite of errors. It can be used to find segments in imperfect transcripts like prompts or closed captions.

\begin{tabular}{|l|l|l|l|l|}
\hline $\begin{array}{l}\text { Radio } \\
\text { station }\end{array}$ & Precision & Recall & F-measure & $\begin{array}{l}\text { Seg. } \\
\text { number }\end{array}$ \\
\hline FrInter & $90.7 \%$ & $96.9 \%$ & $93.7 \%$ & 478 \\
\hline FrInfo & $93.4 \%$ & $89.7 \%$ & $91.5 \%$ & 468 \\
\hline RFI & $98.8 \%$ & $97.8 \%$ & $98.4 \%$ & 812 \\
\hline RTBF & $99.3 \%$ & $97.1 \%$ & $98.4 \%$ & 501 \\
\hline Mean & $96 \%$ & $95.8 \%$ & $95.9 \%$ & 2259 \\
\hline
\end{tabular}

TABLE V

SPOTTING ON THE ESTER DATABASE. EXPERIMENTS ARE PERFORMED ON 3 HOURS OF THE DEVELOPPEMENT SET, BY USING IMPERFECT TRANSCRIPTS OF ABOUT 10\% WORD ERROR RATE. THE RTBF SHOW IS AN EXPERIMENT IN REAL CONDITION

\section{Transcript island Driven decoding}

Finally we have tested the integration of spotting algorithm with DDA. Experiments are performed on 3 hours of the developement set, by using imperfect transcripts of about $10 \%$ Word Error Rate and 50\% of text-segments have been removed for spotting evaluation. The table VI show the DDA search algorithm driven by the perfect transcript and DDA driven by imperfect transcripts.

This set of experiments showed that the spotting algorithm combined with DDA is able to produce a better transcript. Moreover we have the possibility to extract only aligned words. Aligned words are associated with a high level confidence score. Our approach allows us to take advantage 


\begin{tabular}{|l|l|l|l|}
\hline System & Baseline & DDA+IT & DDA+PT \\
\hline INTER & $22.6 \%$ & $17.9 \%$ & $17.1 \%$ \\
\hline INFO & $23.4 \%$ & $21.7 \%$ & $18.3 \%$ \\
\hline RFI & $27.2 \%$ & $23.0 \%$ & $20.3 \%$ \\
\hline Mean & $24.4 \%$ & $20.9 \%$ & $18.6 \%$ \\
\hline
\end{tabular}

TABLE VI

WORD ERROR RATES WITH THE DIFFERENT SYSTEMS; THE BASELINE SYSTEM IS THE STANDARD LIA SPEECH RECOGNITION SYSTEM

(SPEERAL) WHITHOUT HELP, USING ONLY ONE DECODING PASS; $\mathrm{DDA}+\mathrm{IT}=\mathrm{DDA}+\mathrm{IMPERFECT}$ TRANSCRIPT DDA+PT $=$ DDA + EXACT TRANSCRIPT

of all available information: we obtain a better automatic transcription and a better alignment due to the decoder quality. These two criteria increase the size of corpus in only one pass. The search of segments is fast and incremental. In addition experiments with more than one pass show that the system converges to the best potential solution during the first pass.

\section{Results on RTBF in real condition}

The RTBF is a Belgium broadcast news radio. We used 200 hours of speech signal with associated prompts. Prompts are grouped by month and are imperfect. This data allows us to measure the quality of our approach. A language model is estimated on all prompts (about 2400000 words) and merged with a generic language model. Baseline results are presented in table VII from one pass decoding associated with an $a$ posteriori alignment with the text island spotting algorithm: we obtain about 30 hours of exact annotated speech. In a second time we use the transcript island driven decoding. Then the quantity of aligned data is compared. Results show that with the transcript island driven decoding, $38 \%$ of additionnal words are aligned to the prompts: we have 50 hours of exact annotated speech. Moreover, without DDA, spotted segments are similar: the robustness of the spotting island algorithm is retained. These results show significant improvements in the quantity of usable data. The DDA allows us to correct on the fly the ASR system: the increase of data quantity should result in a WER improvement. This experiment brings expected results: a larger corpus with better quality.

\begin{tabular}{|l|l|l|}
\hline System & Baseline & Driven Decoding \\
\hline \# Hours & 200 & 200 \\
\hline \# segments & 50370 & 50370 \\
\hline \# decoded words & 2497125 & 2515503 \\
\hline \# aligned segments & $11158(22 \%)$ & $11487(23 \%)$ \\
\hline \# aligned words & $\begin{array}{l}380042 \quad(15 \%: \\
\text { about } 30 \text { hours })\end{array}$ & $\begin{array}{l}615481 \quad(25 \%: \\
\text { about 50 hours) }\end{array}$ \\
\hline
\end{tabular}

TABLE VII

THIS TABLE SHOW THE NUMBER OF MATCHING WORDS BETWEEN THE

PROMPTS AND THE ASR OUTPUT. THE FIRST COLUMN SHOW THE

BASELINE SYSTEM AND THE SECOND COLUMN SHOW THE TRANSCRIPT ISLAND DRIVEN DECODING METHOD

\section{CONCLUSION AND FUTURE WORK}

We have proposed a method to produce a high quality corpus with a set of recorded audio which have inaccurate prompts and deprived of timecodes. The selection of matching segments showed very good results, while the DDA dramatically improved the decoder quality.

The method was developed in two parts :

- The text island spotting algorithm allows us to synchronize on demand segments with the automatic speech recognition system. The spotting offers a very good Fmeasure (section III-B.3) in real time with large databases of prompts. This allows us to insert the spotting algorithm directly into the decoding process.

- Once the segments are synchronized, DDA is able to correct both prompt and the ouput decoder. As DDA is synchronized only with good segments, the decoding process time is decreased.

The combination of the two proposed algorithms allows us to extract only aligned segments and to correct inaccurate words. This method rapidly produces high quality corpus. Moreover, experiments on the broadcast news RTBF show that the method produces a larger corpus than other a-posteriori approaches. We plan to measure the gains of this approach using the RTBF database for estimate acoustic models.

\section{REFERENCES}

[1] P. Cardinal, G. Boulianne, and M. Comeau, "Segmentation of recordings based on partial transcriptions," INTERSPEECH 2005, 2005.

[2] P. Placeway and J.Lafferty, "Cheating with imperfect transcripts," Spoken Language ICSLP 96. Proceedings, pp. 2115-2118 vol.4.

[3] M. J. Witbrock and A. G. Hauptmann, "Improving acoustic models by watching television," 1998.

[4] P. J. Moreno, C. Joerg, J.-M. V. Thong, and O. Glickman, "A recursive algorithm for the forced alignment of very long audio segments," International Conference on Spoken Language Processing, 1998.

[5] M. Mohri, "Edit-distance of weighted automata," CIAA, 2002.

[6] D. Berndt and J. Clifford, "Using dynamic time warping to find patterns in time series," AAAI Workshop on Knowledge Discovery in Databases, KDD-94, 1994.

[7] P. Placeway, S. Chen, M. Eskenazi, U. Jain, V. Parikh, B. Raj, M. Ravishankar, R. Rosenfeld, K. Seymore, M. Siegler, R. Stern, and E. Thayer, "The 1996 hub-4 sphinx-3 system," Proceedings of the 1997 ARPA Speech Recognition Workshop, pp. 85-89, Feb. 1997.

[8] A. Haubold and J. Kender, "Alignment of speech to highly imperfect text transcriptions," Multimedia and Expo, 2007 IEEE International Conference on, 2007.

[9] H. Chih-wei, "Automatic closed caption alignment based on speech recognition transcripts," 2003.

[10] L. Lamel, J. Gauvain, and G. Adda, "Lightly supervised and unsupervised acoustic models training," Computer Speech and Language, vol. 16, pp. 115-229, 2002.

[11] T. F. Smith and M. S. Waterman, "Identification of common molecular subsequences," J. Mol. Biol. 147, 1981.

[12] D. A. Hull., "Stemming algorithms: A case study for detailed evaluation."

[13] G. Salton and C. Buckley, "Term weighting approaches in automatic text retrieval," Information Processing and Management, 1988.

[14] H. Chan and P. Woodland, "Improving broadcast news transcription by lightly supervised discriminative training," Proceedings of ICSLP, 2004.

[15] L. Nguyen and B. Xiang, "Light supervision in acoustic model training," ICASSP, 2004.

[16] B. Lecouteux, G. Linarès, J. Bonastre, and P. Nocéra, "Imperfect transcript driven speech recognition," in Interspeech'06-ICSLP, Pittburgh, Pensylvania, USA, 2006.

[17] P. Nocéra, G. Linarès, and D. Massonié, "Phoneme lattice based a* search algorithm for speech recognition," Text, Speech and Dialogue : 5th International Conference, TSD 2002, Brno, Czech Republic, 2002.

[18] G. Linarès, P. Nocéra, D. Matrouf, F. Béchet, D. Massonié, and C. Fredouille, "Le système de transcription du lia pour ester-2005," 2005.

[19] B. Lecouteux, G. Linarès, F. Beaugendre, and P. Nocéra, "Text island spotting in large speech databases," Interspeech, 2007. 


\section{BIOGRAPHIES}

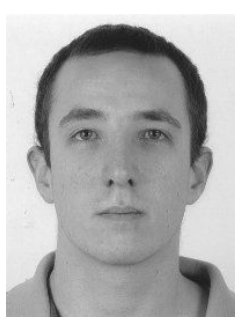

B enjamin Lecouteux obtained a Master in 2005 in the field of automatic speech recognition. He joined the LIA in 2005 as Ph.D. student, in the Speech Processing group. His current research interest are Automatic Speech Recognition and System Combination.

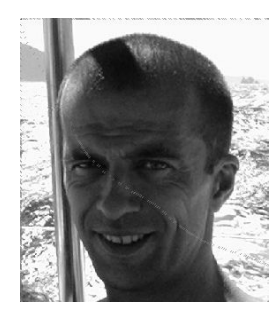

G eorges Linares obtained a Ph.D. in 1998 in the field of Neural networks for acoustic processing. He joined the LIA in 1999 as associate professor, in the Speech Processing group. He participates activelly to the development of the speech recognition system of the LIA (SPEERAL), specially for acoustic modeling and the real time engine. His current research interest are acoustic modeling for Automatic Speech Recognition, decoding strategies, and indexing audiovisual databases. 